\title{
Strategies used to address challenges encountered during website development in South Africa
}

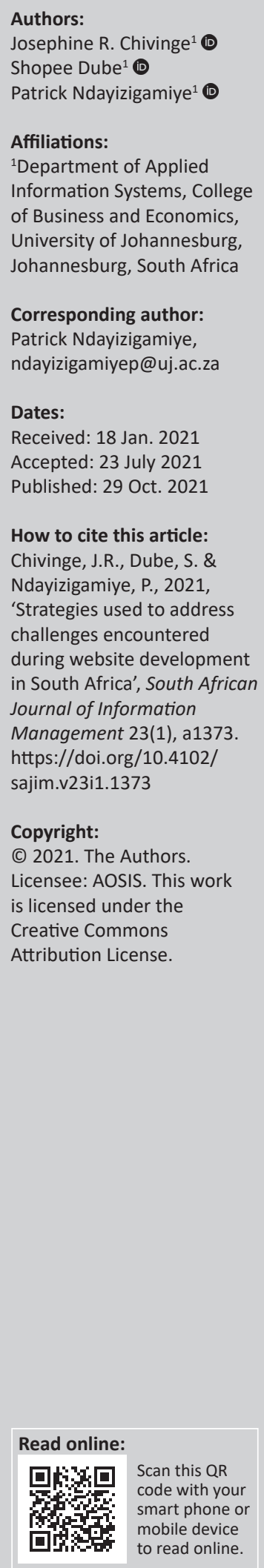

Background: Whether a website design is a simple static page of plain text, a complex e-commerce matrix or a progressive web application, the journey is riddled with challenges. In terms of online users, every second counts when interacting with a website. It is estimated that 2 seconds is the acceptable webpage loading latency that users are willing to tolerate before abandoning a webpage. Users expect web applications to be more usable, reliable, secure, personalised and context-aware.

Objectives: This study sought to identify the challenges faced and the strategies used during website development, and to then map a conceptual framework to address these challenges.

Method: The 7Cs framework was the theoretical framework underpinning the study. Based on qualitative research, 12 website developers were sampled in Gauteng Province, South Africa, using non-probability sampling methods.

Results: The 7Cs Website Development Scheme (7CWDS) charted from the research findings shows that the following strategies are essential in overcoming website development challenges: (1) good communication between developer and client, (2) agile planning and acceptance of project scope, (3) creation of website drafts and acceptance, (4) flexible website designs, (5) agile website development using a step-by-step approach, (6) website connections to Google Analytics, (7) connecting website to social media and (8) website cybersecurity scanning and testing.

Conclusion: The study developed the 7CWDS as a toolkit that underlines critical strategies to address common challenges in website development, and presents them in a logical hierarchy and order of execution.

Keywords: Website development; 7Cs framework; Website development challenges; Website development strategies; 7Cs Website Development Scheme; 7CWDS.

\section{Introduction}

In this competitive age, business organisations must strategise and appreciate the wideranging benefits of website development initiatives to streamline their workflow, reduce cost and expand their customer base (Knoke 2018). Website developers face several challenges when developing websites. Therefore, they are required to implement several strategies to ensure that the website development process is successful. When clicking on websites links, users often do so with the intent of obtaining information. They stay on the website longer if the website performs the functions related to obtaining information easily, for example, being able to search a product by category on an e-commerce website. Thus, it is vital to strategically display important information on the website and develop user-friendly interfaces. In terms of online users' interaction with a website, every second counts. On what is the acceptable webpage loading latency, Rempel (2015) reported that users are willing to wait an average of 2 seconds before abandoning the page. Users expect web applications to be more usable, reliable, secure, personalised and context-aware (Rempel 2015).

Website development solutions include website accessibility from different types of devices (Rab 2018). Sharma and Lijuan (2015) found that information and online service quality are crucial to user satisfaction. A user's intention to maintain a connection with a website depends on the perceived benefits of good quality service and trust (Sharma \& Lijuan 2015). Their study concluded that good quality websites tend to attract potential customers, encourage new buyers and retain repeat customers. Aakash and Aggarwal (2019) came to a similar conclusion that high website quality, user satisfaction and online user feedback reviews increase the prospects of online shopping businesses to make a profit. 
Whether a website design is a simple static page of plain text, a complex e-commerce matrix or a sophisticated progressive web application, the design journey is not always a smooth ride. Studies have revealed a wide range of challenges and strategies used in website development (Dundu 2017; HungJoubert \& Erdis 2019; Katuu 2018; Mathaba \& Mkhize 2018; Mujinga, Eloff \& Kroeze 2018; Ogutu 2016). These studies dealt with challenges in website development, namely, website cybersecurity and trustworthiness, internet penetration and mobile platforms, website usability, accessibility and assessment, linking websites to social media websites and website 7Cs quality elements.

In South Africa, challenges encountered by website developers have not been widely investigated. Therefore, this study sought to identify the challenges faced by website developers in South Africa, to determine the strategies they currently use to resolve such challenges, and to map a conceptual framework to address these challenges encountered during website development in the South Africa's context.

The study developed the 7Cs Website Development Scheme (7CWDS) as a toolkit that underlines critical strategies to address common challenges in website development, and presented them in a logical hierarchy and order of execution.

\section{Literature review}

\section{Factors that influence website visits}

Challenges related to website cybersecurity threaten all users, and website developers have to strategise how to alleviate security and privacy concerns during online transactions (Mathaba \& Mkhize 2018). Using a convenience sampling approach, Mathaba and Mkhize (2018) investigated perceptions of users of online shopping. They found that websites that deliver on their promises and convey website credibility are likely to be trusted, and such trust reduces the perceived risks generally associated with online shopping (Mathaba \& Mkhize 2018). In the South Africa's context, Mujinga et al. (2018) showed that only $4 \%$ of South Africans with bank accounts use online banking. Some of the reasons given for this trend are the concerns about identity theft and internet fraud, with South Africa ranked third on reported cyber-attacks in the world, as a result of the unpatched systems (Van Heerden, Von Solms \& Vorster 2018). Van Heerden et al. (2018) suggested relevant cybercrime education and awareness interventions for website developers to address website vulnerability. These researchers further identified the need for further research on how to reduce fundamental website risk factors (Van Heerden et al. 2018).

Device preference of online banking users was investigated by Mujinga et al. (2018), and they concluded that website usability, device compatibility and web-security goals are critical in drawing new web visitors and have a direct impact on client satisfaction. In the unfortunate event of a successful hacking exploit, it may be more costly to recover from it, than the cost of implementing strategic preventative measures.
Studies by Mbise et al. (2018), Mujinga et al. (2018) and Robb and Hawthorne (2019) shared the view that the prospects of a successful internet-based (web-based) business depend on various factors including internet penetration, data costs and the demographics of the target market. Robb and Hawthorne (2019) suggested the rollout of high-speed fibre optic network (which has recently been introduced in South Africa) as a possible solution to the connectivity and penetration issues. Based on these studies, one may deduce that there is a need for website developers to: (1) position their products favourably, (2) find and gear-up to the trends in internet data services and, (3) consider the necessity for mobile device compatibility and produce superior website usability quality across devices.

The consequences of having a poor website negatively impacts a business it is supposed to promote. Several studies (Mujinga et al. 2018; Iram, Zafar \& Zahra 2018; Verkijika \& De Wet 2018) agree on the view that while the developer may be satisfied after developing a website, the users may have negative perceptions about the website usability. Iram et al. (2018) argued that website developers should consider the degree to which a website is readable, functional and usable from the perspectives of the average user. Some of the aspects of website usability that need to be considered are: word or sentence complexity, faint font colours and the use of capital letters (Iram et al. 2018). Website usability is the extent to which a website may be used without frustration, difficulty, hesitation or questions (Mujinga et al. 2018). While the global average time spent on a webpage is 2 minutes, users tend to exit a webpage that they find less engaging within the first 15 seconds, and abandon webpages that fail to load in 2 seconds (Ullah, Sepasgozar \& Wang 2018).

Various studies have investigated website usability using few evaluation tools (Domínguez Vila, Alén González \& Darcy 2018; Katuu 2018; Oyefolahan et al. 2019). Katuu (2018) evaluated the Mandela Portal website using Google Analytics and was able to identify visitors' geographic origins to formulate strategies on content improvements. The degree of accuracy of the evaluation depends on the type of usability evaluation tool used as each tool provides its own set of scores. The automated assessment embedded within these evaluation tools determines the rating of internal quality attributes of the website especially the size of HTML pages, image sizes, file formats, links, compatibility with browsers, download and load time, compatibility with mobile devices, page ranking and other technical deficiencies within a website (Kaur, Kaur \& Kaur 2016).

\section{Linking websites to social media websites}

Social media can be useful for marketing products, pursuing strategic goals, and promoting a company's reputation. In social networking platforms, people are proud to be associated with specific brand names and they tend to post the same on their Facebook (Dundu 2017). Social media can raise the buyer's trust by $51 \%$ and the intention to transact by $44 \%$ (Ullah et al. 2018). The extent to which trust in a brand is spread through a social media network depends 
mainly on the size of the social media network. Dundu (2017) proposed some strategies on how website developers may foster trust in their website. These include using attractive web-design, continually updating and refreshing web-content, adding pictures and videos for a human-feel, adding links to other social media websites, ensuring short webpage loading time, including the business address on the website, proofreading the website content to remove errors and adding a feedback or comment page.

From the above literature, it is evident that a website developer deals with many challenges. The way a developer handles these challenges can result in visitors being attracted to the website, alternatively it can drive them away (Van den Heever \& Rensburg 2018).

\section{The 7Cs conceptual framework}

The study adopted the 7Cs framework as the theoretical framework underpinning this research. Developed by Rayport and Jaworski (2002), the 7Cs framework aims to standardise the development of e-commerce websites. The 7Cs framework comprises specific elements, namely: (1) context which focusses on the layout and overall website design, (2) content focussing on textual and visual design, (3) community which is the level of interaction with customers, (4) customisation being the website's ability to adapt according to user's preferences, (5) communication which entails facilitating the user-to-vendor online communication, (6) connection that the website has with other websites and, (7) commerce as the ability of the website to complete online transactions.

A study by Ogutu (2016) identified the particular elements linked to a website's perceived usefulness and ease-of-use. He found that most users were satisfied with the community element that provided chat pop-ups, the content that assured the information on the website is continuously up-to-date, and the customisation of the individual viewing experience (Ogutu 2016). Most users also liked the communication element that provided quick automated email responses and the connection element that linked the banking websites to other websites (Ogutu 2016). The limitation of Ogutu (2016) study was that data collection was performed through questionnaires, and he acknowledged the difficulty of telling how truthful the respondents were.

This study adopted the 7Cs framework as the underpinning theoretical framework. In exploring the challenges encountered in the quest to comply with the 7Cs elements, this study used the qualitative research method with face-to-face interviews to gain deeper understanding of the challenges encountered during website development and the strategies used to address them within the South African context. The study's research objectives sought to identify the challenges and strategies in each element of the 7Cs framework as follows:
1. Context: to identify challenges and strategies with the website layout

2. Content: to identify challenges and strategies when gathering website content

3. Commerce: to identify challenges and strategies when developing e-commerce websites

4. Community: to identify challenges and strategies when integrating a website with social media

5. Customisation: to identify challenges and strategies while developing customisation options

6. Communication: to identify challenges and strategies when requesting feedback from the user

7. Connection: to identify challenges and strategies when linking website to website.

\section{Research design and methods}

This study used the interpretivist philosophy and deduction as the research approach. It used the interpretivism philosophy to understand web developers' everyday work experiences and their perceptions about the challenges encountered during website development.

This study employed the qualitative research method, using a set of 15 open-ended interview questions (Tracy 2019). The interview questions were formulated based on an extensive literature review on the challenges and strategies pertaining to website design. The target population for this study was experienced website developers operating in Gauteng province of South Africa, with an average of 9 years of experience. Non-probability sampling methods, namely convenience sampling coupled with snowball sampling method, were used to identify and select the participants in this study. Convenience sampling identified the initial research participants. Then the participants were requested to refer the researchers to other potential participants. The sample size consisted of 12 respondents. Interviews were audio-recorded and transcribed using the Amazon Transcribe web application (Matheson 2007). Each interviewee was assigned a specific code (from [P1] to [12]) to maintain the anonymity of the research participants. In this paper, the participants are referred to by their codes. Each interview question was mapped to a corresponding element of the 7Cs framework. In addition, each response was coded and matched with the corresponding research question. Moreover, thematic analysis was used to generate themes that were aligned with the 7Cs framework. Not all the interviewees' responses are captured in this paper because of the page limitation of a journal article.

\section{Results and discussion}

The demographics of the respondents included the respondents' level of education, position in the company, years of experience as a website developer and the types of websites the respondent's business develops. Eleven participants were degree holders, highly experienced in programming and 
website development because of their years of experience. Only one participant had a diploma qualification. The participants were website developers from businesses serving different core businesses from banking to e-commerce.

The findings were organised according to the research questions and grouped according to the emerging themes. The themes that emerged were subsequently grouped according to the elements of the 7Cs framework. For ease of analysis, each response related to a challenge in website development was paired with the corresponding strategy where applicable.

\section{Context: Challenges and strategies related to website layout}

In line with the research objectives of the study, respondents were required to indicate the challenges they encountered with website design layout and the strategies used to address the challenges, respectively. One participant (P2) said:

'Challenge in ensuring corporate standards are maintained in the graphic designs of the logo' and the strategy was 'Clients to provide graphic images and photographs, redrawing may be required, a web developer has to learn graphic design skills.' (P2, Degree in Computer Science, 15 years of work experience)

A lot of time is wasted on issues around the context element of website layout. There are often misunderstandings between designers who at times present their ideas in Photoshop Document Design (PDD) format and the website developers who require more specific details to code, including the background colour, font type and font size (Hadar et al. 2018). One participant (P3) said:

'I hate graphic designers! They think I can draw a triangle here, draw a logo there, put text here with the click of a button, or move the image around just like that. It is about coding, and it takes time.' (P3, B. Com IT Systems Development, 5 years of work experience)

Another participant (P6) expressed that:

'[There are] challenges in understanding the client's design layout, when client's preferences conflict with reasonable design standards, or when a client does not have any input because they do not know what they want.' (P6, Diploma in Business Information Technology, 5 years of work experience)

The strategy proposed was:

'Use the standards on design structure and layout, provide the client with multiple suggestions for the choice of design layouts. Use a design that is flexible to changes when necessary.' (P6, Diploma in Business Information Technology, 5 years of work experience)

Participants (P1 and P3) also mentioned that clients often changed their minds about their requests. Thus, they suggested strategies to deal with these problems, such as using an information request form that incorporates essential details like background colour, font type and font size. They recommended that the website developer could incorporate a maximum change statement in the contract that limits the number of free changes that could be requested by the client to three (3). It seems there was a consensus on the view that the website developer would keep the work-scope to design requests, use web designer graphics, start with a wireframe and submit the wireframe for approval to ensure the client is satisfied with the design and layout before proceeding. In terms of the use of wireframe, Robinson (2019) suggested taking the approved wireframe, and then add required specifications to make a mock-up, which, when accepted, is developed into a fully functional website prototype for demonstration.

\section{Content: Challenges and strategies related to gathering website content}

It was found that too much or too little information can cause problems with the content element of the 7Cs framework. For example, the website layout design may not accommodate the content, with the text overlapping to other sections, or the client could provide insufficient content to suit market objectives. A participant (P3) proposed, 'If there is overlapping text on a section, we can add a "read more" link that takes the user to another page'. According to the same participant (P3):

'The challenge might arise from the website layout, not being able to accommodate the website content. As a result, some text might overlap the designed sections or may not look presentable.' (P3, B. Com IT Systems Development, 5 years of work experience)

Various strategies to respond to the challenge were proposed by some participants ([P1] and [P3]):

'To combat the design-text overlap challenge, we make sure that we build the website as flexible as possible ... to ensure that when the client fills in the content the design and layout of the website is not spoiled. We do this by using the Bootstrap 4 Framework that enables us to use rows and columns that can collapse depending on the browser screen width and the content within the elements.' ([P1, Masters in Humanities, 20 years of work experience] and [P3, B. Com IT Systems Development, 5 years of work experience])

Similarly, Luong (2019) suggested fixing overlapping content with the Bootstrap 4 framework that provides flexible layouts and compatibility with mobile devices.

Respondent (P3) also complained about clients who would provide content information with typing errors:

'The other challenge is that clients expect us to proofread the website content to ensure that there are no grammar or spelling errors. This can be very challenging for a developer as it's not our area of speciality.' (P3, B. Com IT Systems Development, 5 years of work experience)

The strategy proposed was to communicate with the client via the contract that proofreading of the website content is not performed; the client should provide accurate content.

\section{Commerce: Challenges and strategies related to developing e-commerce websites}

Participants reported that the commerce element in e-commerce websites came with many functionalities that 
make the website complicated. For example, website developers must deal with WooCommerce add-ons that may not integrate with shipment classes or payment gateway options which may affect the functionality of the rest of the website. A participant (P1) said, 'If a different shipping method or different payment gateway is used, then it has effects on how the rest of the website operates'. Some other challenges mentioned in relation to maintaining an e-commerce presence included: choosing specific plug-ins for payment gateways, selection of shipping classes, customising the website for multiple search engines that drive traffic and the clients' lack of trust in the website developers and servers when it comes to creating the e-commerce functionality. Participant (P1) further mentioned:

'Most of our clients think of an e-commerce website as a simple website while it's a website application... So, normally, our challenge is to communicate that what we're doing is very complicated, but a client often thinks it's straightforward. ... the biggest obstacle for e-commerce in South Africa is delivery. It's complicated to deliver to people's houses. Plus, e-commerce in South Africa in most cases works for the top $20 \%$ of the population with street addresses .... (P1, Masters in Humanities, 20 years of work experience)

\section{Participant (P6) also mentioned that:}

'Building an e-commerce website can be tasking and resourcedemanding. Clients want something reliable and not prone to hacking and other security breaches. The challenge is to make this cost-effective at the same time.' (P6, Masters in Humanities, 20 years of work experience)

In terms of strategy, a participant (P6) mentioned the use of the Sandbox testing tool.

\section{Community: Challenges and strategies related to integrating a website with social media}

The community element of social media comes with many challenges of regulations. To limit what is accessible, the developer may create a dedicated separate account for website feed linking to social media platforms. A participant (P12) noted:

'Most companies are not present on social media, so if their websites have to be linked, the developer has to register and create their pages on the social media platforms.' (P12, BSc Computer Sciences Honours, 10 years of work experience)

Another participant (P3) discussed the branding of social media widgets in the major colours of the company logo:

'At times, they want to display social media feed on their website. However, it's not easy to customise the design of the social media feed widget, because the plugin is usually provided by the social media platform in a format that is not editable ...' (P3, B. Com IT Systems Development, 5 years of work experience)

An online presence on social media has to be controlled and well managed to maintain company brand quality. A participant (P4) described a strategy that entails sharing only selected pages of a website approved for public distribution: 'We section website content and give each section page a unique tag. Only the company approved pages can be shared'. Kapoor et al. (2018) echoed this finding that social media is propelled by user-generated content, and therefore, requires suitable network setting configurations, to be able to influence purchasing or selling behaviours and political agendas.

\section{Communication: Challenges and strategies related to requesting feedback from the user}

The communication element covers issues about requesting feedback from the website user.

A popular communication link with instant feedback is a chat system installed on a website using the Auto Chat Live plug-ins. A live chat system allows website visitors to seek information from a business through its website (McLean \& Osei-Frimpong 2017). The live chat platform has an administration side, where responses to the customer's request are generated. Participant (P3) mentioned that there are no challenges associated with the live chat platform, but rather with feedback forums: 'Remember, forums are open to everyone. Some customers can be rude, and they write on the forum to tarnish your name' (P3). In terms of strategy, (P3) suggested an approval system to regulate customer feedback:

'The corrective strategy is channelling all writing on the blog forums via an administrator to approve. When the administrator does not approve, customers don't know their posts fail to display because of human intervention, they think webpage has errors. They end up reporting website page errors unnecessarily.' (P3, B. Com IT Systems Development, 5 years of work experience)

\section{Customisation: Challenges and strategies while developing customisation options}

The customisation element of the 7Cs framework facilitates the development of a website that is useful for people with different skills, needs and preferences and includes people with disabilities (Kous \& Polančič 2019). Customisation challenges often arise when the developer has to make the site flexible using a compatible plug-in within a specific budget and project timeframe. One participant (P4) said:

'Most customisation options are provided by themes and website development platforms through the use of plugins. The challenge is that at times, the plugins are not accurate or not fully functional. Our strategy is to test the customisation functionality.' (P4, Bachelors degree in Information Technology, 1 year of work experience)

The proposed strategy was to 'test the customisation functionalities repeatedly while making adjustments' (P4). Useful customisation should be tailored to the preferences of the target market to minimise user frustrations and eliminate challenges experienced by most people interacting with the website (Kous \& Polančič 2019). 


\section{Connection: Challenges and strategies related to linking a website to another website}

The connection element of the 7Cs framework is considered when linking a website to another website. Application Programming Interface (API) brings in the desired functionality, actions and communication protocols from one website into another, so that the developer does not need to reinvent the code (Sawant 2019). A participant (P3) suggested:

'We need first to understand the API requirements so that we pass through all the necessary information to obtain the data that we need from the other website. At times we must convert request data to a particular format, for example, from JSON to Swagger.' (P3, B. Com IT Systems Development, 5 years of work experience)

However, using API features can bring bugs into the accepting website code (Sawant 2019). Participants said generally all bugs can be fixed, but doing so takes time. The challenge then is to answer the question: when does a developer give up on a bug fix? A participant (P7) responded:

'For me, I am stubborn. I can stay on a problem for a week if the timeframe allows. But for others, I can say maybe a day or two, and they call for help.'

In terms of strategy, participant (P7) indicated that it is necessary to suggest possible options to the client whenever the website developer was stuck. Participant (P7) further mentioned:

'... when you fail, you go back to your client and say "I can't do that, but I can do this." Always go to the client with a form of a solution.' (P7, Bachelor's Degree in Computer Science, 10 years of work experience)

Moreover, generally, integration is a high project timeconsuming item. What often happens is that designed functionality is mistaken for a bug issue. The strategies suggested by the respondents to counter this challenge included referring to proper documentation, implementing end point to end point security to eliminate threats and using APIs that offer programming support. The APIs should use standards widely expected, allowed, inferred and tested (Sawant 2019).

A website needs testing to ensure it is secure and functions as desired. A website must be reliable and shielded from security breaches. Participant (P1) noted:

'The website code has to be secure. Outside penetration testing is required. That cannot be a one-person job. We need many brains to see who [one] can break into a website.' (P1, Masters in Humanities, 20 years of work experience)

The challenge is to make every code safe and functional while remaining cost-effective. Binkowski, Appel and Assmuth (2018) recommended the Sandbox testing tool to test the functionalities of e-commerce website before the site is officially deployed.
Throughout the interviews, the key strategies that participants kept stressing as being fundamental to proper website development were noted. A review of all the findings, key strategies and the sequence of their application was central to developing the conceptual framework. The hierarchical list starts with agile planning at the top and ends with website cybersecurity at the bottom.

The conceptual framework developed from the findings is referred to as the 7CWDS, in recognition of the 7Cs framework on which it is based, and is presented graphically in Figure 1.

\section{The 7Cs Website Development Scheme}

Several studies have concurred on the need for good communication in website development projects (Mathaba \& Mkhize 2018; Sousa \& Rocha 2019). On top of the ladder, the framework introduces agile planning. The agile planning is the step-by-step flexible planning of short cycles of tasks, approved through effective two-way communication between the developer and the client. The 7Cs framework breaks down the desired website quality into seven deliverable elements. Similarly, a website development project can be broken down into self-contained tasks or sprints that can be easily managed from start to finish.

The next step is the creation of a website draft, often starting as a handwritten paper sketched wireframe. A wireframe outlines the basic structure showing major design elements, but may not include detailed specifications such as colours (Robinson 2019). The wireframe is then reviewed, approved and any required specific details added; by then, it is called a mock-up (Robinson 2019). When the mock-up design is finalised and accepted, the developer translates it into executable codes, making a digital representation often called a prototype. The prototype serves to demonstrate specifications in the form of a real, working website rather than an abstract construct (Da Silva Ferreira 2019).

In step 3, the use of Bootstrap 4 is the common strategy chosen as a website development platform to make flexible website design that assists in mobile responsiveness (Luong 2019). The actual choice of website development software depends on the preferences and experience of the developer. A developer must, however, choose the most suitable templates and plug-ins carefully.

At step 4 - down the ladder, the framework features agile website development as a means to deliver superior performance, scalability and security in a short timeframe regardless of continuous changes the customer may request. Agile website development is a development methodology that allows for flexibility in responding to changes when a client alters specifications, easy adaptability and reducing development time by streamlining project tasks (TorrecillaSalinas et al. 2015). The methodology ensures that the developer does not derail or move many steps backwards when the client proposes changes. 


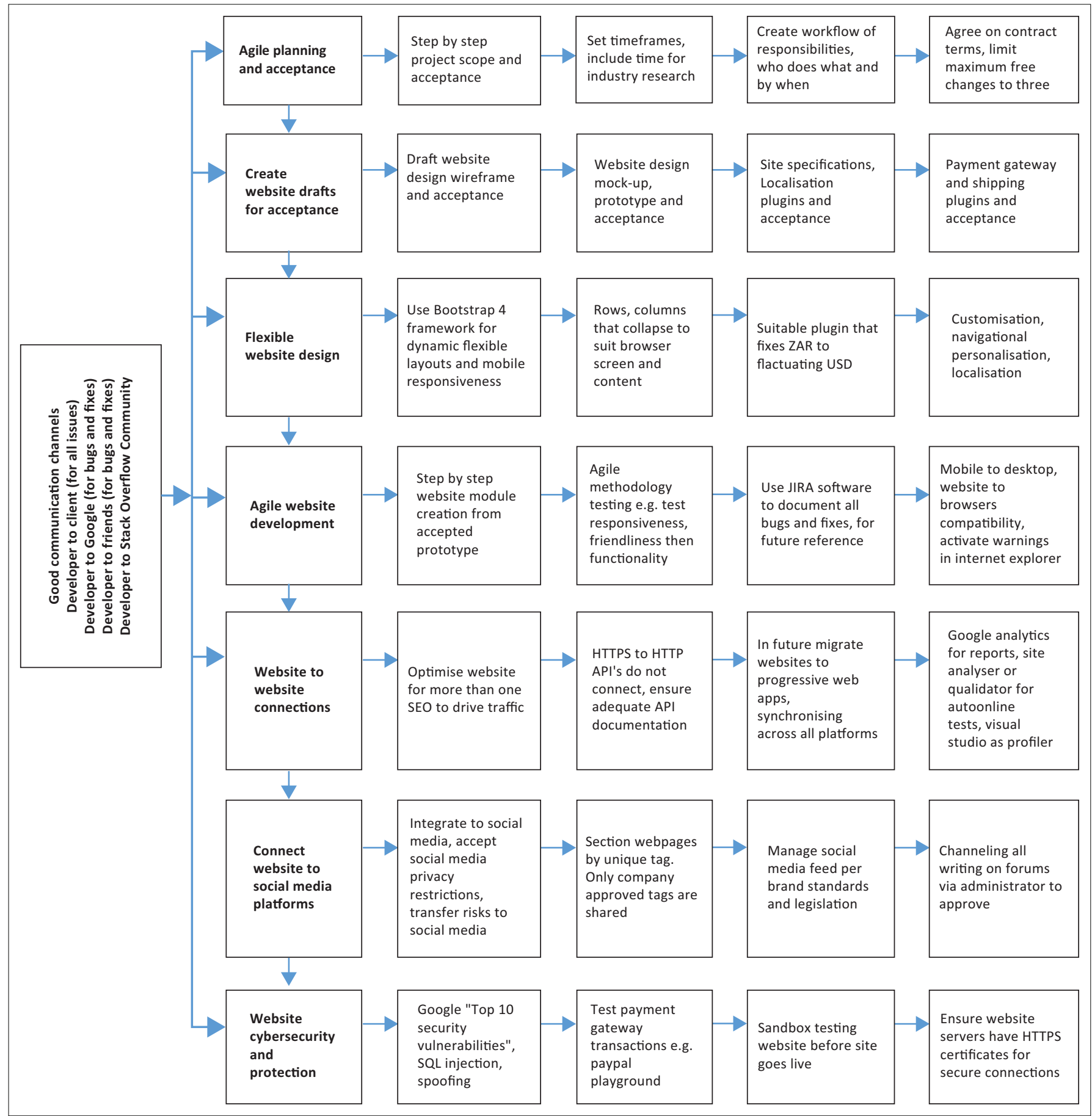

API, Application Programming Interface; SEO, Search Engine Optimisation.

FIGURE 1: 7Cs Website Development Scheme.

In step 5, the focus is on connection - an important element of the 7Cs framework with special pre-requisites before linking websites to other websites. As a good practice, website developers should be careful not to create links to websites that promote illicit matter, cyberbullying, illegal downloads or invade privacy. Developers, seeking highest rankings in search engine results consider the factors that contribute to the search engine algorithm rankings namely, HTTPS connection, website quality, social media support, keywords in the title tags, structure of the website, size of the website, loading time, domain age and keyword density (Ziakis et al. 2019). The connection of a website to other website(s), as indicated in the framework, is, therefore, valuable.

Another connection feature in the 7CWDS is the integration with a wide range of social media platforms (step 6), namely the community element. Connecting websites to social media platforms helps increase traffic to the website and provides the quality backlinks that increase website trustworthiness (Ziakis et al. 2019). The challenge of controlling what content can be shared or cannot could be solved by tagging webpages with unique numbers, and then sharing the company approved tags. 
Website cybersecurity and protection issues appear at the bottom of the framework (step 7). Its position in the framework is supported by Binkowski et al. (2018) who insisted on Sandbox testing of all website functionalities and cybersecurity at the end of development, but before the website goes live. In this step, a website developer should be acquainted with the most common vulnerabilities, test payment gateway(s) for possible vulnerabilities, use Sandbox testing before a website goes live and ensure that website servers have HTTPS certificates for secure connections.

\section{Limitations}

The focus of the study was on expert knowledge and challenges and interventions or strategies provided by experienced website developers employed in South African businesses. The research acknowledges that there could be other challenges and strategies that were not investigated in the study.

The study was limited in time and design scope as it focussed only on website development challenges faced by website developers in Gauteng province of South Africa. This study recommends that research involving other types of business website/s can assist with conceptualising and designing further related studies in other geographical locations.

Lastly, further empirical research using both quantitative and qualitative methods could be performed to test the effectiveness of the 7CWDS scheme developed from this study's findings.

\section{Implications and recommendations}

Pflügler et al. (2018) argued that challenges could present opportunities to learn and avoid repeating the same mistakes. Seth, Bagalkoti and Nigam (2019) used the Jira software to document all bug fixes and predict timelines to fix future bugs. This study found that website developers were using less code and more plug-ins. This study, therefore, recommends using plug-ins and small code adjustments to achieve the desired functionality and device compatibility (Liarokapis et al. 2018).

\section{Conclusion}

This article focussed on identifying the challenges faced during website development, determine the strategies used and map a conceptual framework to address them. The 7Cs framework was the theoretical framework underpinning the study. The qualitative research method was employed in this study. Interviews were conducted with 12 website developers using 15 open-ended questions, which were formulated based on an extensive literature review.

The 7CWDS framework charted from the research findings stands on: (1) agile planning and acceptance of project scope, timeframes and the terms of the contract, (2) creation of website drafts and acceptance of wireframes, mock-ups and prototypes, (3) flexible website designs using dynamic and responsive layouts, (4) agile website development using a step-by-step approach, (5) website connections for Search Engine Optimisation (SEO) with secure HTTPS, (6) connecting websites to social media platforms while managing what can be shared and published and, (7) website cybersecurity scanning and testing.

All these steps are anchored on the need to establish good and secure communication channels across all stakeholders in website development process. The 7CWDS is recommended as a possible toolkit to address challenges concerning website development. It highlights the crucial strategies needed to address the significant challenges found in this study and presents them in a logical hierarchy that suggests the order of execution. Website designers, developers and website owners can benefit from using this toolkit as it gives insight into possible improvements in website development, and suggests how these might be achieved. Businesses may choose which aspect(s) of the 7CWDS framework to adopt and how to adopt them, and it can also be beneficial to the private sector businesses in South Africa and other countries, keen to understand how effective website development can profit their businesses.

\section{Acknowledgements}

The authors would like to thank all the participants in the study.

\section{Competing interests}

The authors have declared that no competing interest exists.

\section{Authors' contributions}

S.D. and P.N. conceived the study and designed the data analysis. J.C. collected the data, performed the analysis, and wrote the first draft of the article. P.N. contributed data analysis tools and edited this article.

\section{Ethical considerations}

The study protocol was approved by the ethics committee in The School of Consumer Intelligence and Applied Information Systems at the University of Johannesburg. The ethics approval number is: 2020SCiiS05.

\section{Funding information}

This research received no specific grant from any funding agency in the public, commercial or not-for-profit sectors.

\section{Data availability}

The data that support the findings of this study are available on request from the corresponding author, P.N. The data are not publicly available as they contain information that could compromise the privacy of the research participants. 


\section{Disclaimer}

The views and opinions expressed in this article are those of the authors and do not necessarily reflect the official policy or position of any affiliated agency of the authors, and the Publisher(s).

\section{References}

Aakash, A. \& Aggarwal, A.G., 2019, 'Role of EWOM, product satisfaction, and website quality on customer repurchase intention', in I.C.D.A. Carvalho \& E.M.C.B. Sabino (eds.), Strategy and superior performance of micro and small businesses in volatile economies, pp. 144-168, IGI Global, Hershey, PA.

Binkowski, C., Appel, S. \& Assmuth, A., 2018, 'Securing 3rd party app integration in docker-based cloud software ecosystems', Cloud Computing 2018, 77-83.

Da Silva Ferreira, J.C., 2019, 'Live web prototypes from hand-drawn mockups', Masters dissertation, Universidade do Porto, Porto.

Domínguez Vila, T., Alén González, E. \& Darcy, S., 2018, 'Website accessibility in the tourism industry: An analysis of official national tourism organization website around the world', Disability and Rehabilitation 40(24), 2895-2906. https://dol. org/10.1080/09638288.2017.1362709

Dundu, N., 2017, 'Factors that influence students' engagement with the Facebook page of a selected University', Doctoral dissertation, University of Johannesburg.

Hadar, I., Hasson, T., Ayalon, O., Toch, E., Birnhack, M., Sherman, S. et al., 2018 'Privacy by designers: Software developers' privacy mindset', Empirical Software Engineering 23(1), 259-289. https://doi.org/10.1007/s10664-017-9517-1

Hung-Joubert, Y.T. \& Erdis, C., 2019, 'Influence of retailers' website system quality factors on online shopping in South Africa', African Journal of Science, Technology, Innovation and Development 11(2), 211-221. https://doi.org/10.1080/20421338. Innovation and

Iram, N., Zafar, S. \& Zahra, R., 2018, 'Web content readability evaluation using fuzzy logic', in 2018 International Conference on Advancements in Computational Sciences (ICACS) proceedings, Lahore, Pakistan, February 19-21, 2018, pp. 1-8.

Kapoor, K.K., Tamilmani, K., Rana, N.P., Patil, P., Dwivedi, Y.K. \& Nerur, S., 2018, 'Advances in social media research: Past, present and future', Information Systems Frontiers 20(3), 531-558. https://doi.org/10.1007/s10796-017-9810-y

Katuu, S., 2018, 'Using web analytics to assess traffic to the Mandela Portal: The case of African countries', New Review of Information Networking 23(1-2), 1-18. https://doi.org/10.1080/13614576.2018.1523741

Kaur, S., Kaur, K. \& Kaur, P., 2016, 'Analysis of website usability evaluation methods', in 3rd International Conference on Computing for Sustainable Global Development (INDIACom) proceedings, New Delhi, India, March 16-18, 2016, pp. 1043-1046.

Knoke, D., 2018, Changing organizations: Business networks in the new political economy, Routledge, New York.

Kous, K. \& Polančič, G., 2019, 'Empirical insights of individual website adjustments for people with dyslexia', Sensors 19(10), 2235. https://doi.org/10.3390/s19102235

Liarokapis, D., Papigiotis, V., Stergiou, E. \& Gogos, C., 2018, 'Creating an electronic magazine in WordPress for promoting the practical training of students', in 2018 International Conference on Information Technologies (InfoTech) proceedings, International Conference on Information Technolog
Varna, Bulgaria, September 20-21, 2018, pp. 1-4.

Luong, Q., 2019, 'Web application development with Reactjs framework', Doctoral Thesis, University of Applied sciences, Information Technology, Vaasa.

Mathaba, R. \& Mkhize, N., 2018, 'Exploring the relationships between antecedents of online purchasing behavior', Management Dynamics 184.

Matheson, J.L., 2007, 'The voice transcription technique: Use of voice recognition software to transcribe digital interview data in qualitative research', The Qualitative Report 12(4), 547-560.

Mbise, T., Taal, S., Roberts, M. \& Lammersen, F., 2018, Digital connectivity \& e-commerce: Overview of financing flows and examples of aid for trade support World Trade Organization (WTO), Geneva.
McLean, G. \& Osei-Frimpong, K, 2017, 'Examining satisfaction with the experience during a live chat service encounter - implications for website providers', Computer in Human Behavior 76(C), 494-508. https://doi.org/10.1016/j.chb.2017.08.005

Mujinga, M., Eloff, M.M. \& Kroeze, J.H., 2018, 'System usability scale evaluation of online banking services: A South African study', South African Journal of Science 114(3-4), 1-8. https://doi.org/10.17159/sajs.2018/20170065

Ogutu, R., 2016, 'Factors of user interface design that influence usage of e-banking websites', Masters dissertation, University of KwaZulu-Natal, Durban.

Oyefolahan, I.O., Sule, A.A., Adepoju, S.A. \& Babakano, F., 2019, 'Keeping with the global trends: An evaluation of accessibility and usability of Nigerian banks websites', International Journal of Information Engineering and Electronic Business 11(2), 44-53. https://doi.org/10.5815/ijieeb.2019.02.06

Pflügler, C., Malzer, T., Jäschke, T., Wiesche, M. \& Krcmar, H., 2018, “'Do I want to have losers in my team?" A quantitative study of learning from IT project failure', in Proceedings of the 51st Hawaii International Conference on System Sciences, Hawaii, USA, January 2-6, 2018, pp. 4348-4357.

Rab, S., 2018, 'A university website using Content Management System (CMS) and add-on plugins', Doctoral dissertation, United International University, Dhaka, Bangladesh.

Rayport, J.F. \& Jaworski, B.J., 2002, Introduction to e-commerce, McGraw-Hill, Boston.

Rempel, G., 2015, 'Defining standards for web page performance in business applications', in Proceedings of the 6th ACM/SPEC International Conference on Performance Engineering, Austin-Texas, USA, 28 January-04 February 2015, pp. 245-252.

Robb, G. \& Hawthorne, R., 2019, 'Net neutrality and market power: The case of South Africa', Telecommunications Policy 43(9), 101814. https://doi.org/10.1016/j. telpol.2019.03.003

Robinson, A., 2019, 'Sketch2code: Generating a website from a paper mockup', Undergraduate dissertation, University of Bristol, Bristol.

Sawant, A.A., 2019, 'The impact of API evolution on API consumers and how this can be affected by API producers and language designers', Doctoral dissertation, Delft University of Technology, Delft.

Seth, S., Bagalkoti, V. \& Nigam, M.J., 2019, JIRA report extraction, Jaypee University of Information Technology, viewed 23 April 2020, from http://ir.juit.ac.in/ 123456789/22908.

Sharma, A.P. \& Sharma, S., 2018, 'Risk management in website development', in Analyzing the role of risk mitigation and monitoring in software development, pp. 188-203, IGI Global, Hershey, PA.

Sharma, G. \& Lijuan, W., 2015, 'The effects of online service quality of e-commerce websites on user satisfaction', Electronic Library 33(3), 468-485. https://doi. org/10.1108/EL-10-2013-0193

Sousa, M.J. \& Rocha, Á., 2019, 'Skills for disruptive digital business', Journal of Business Research 94, 257-263. https://doi.org/10.1016/j.jbusres.2017.12.051

Torrecilla-Salinas, C.J., Sedeño, J., Escalona, M.J. \& Mejías, M., 2015, 'Estimating, planning and managing agile website development projects under a value-based perspective', Information and Software Technology 61, 124-144. https://doi. org/10.1016/j.infsof.2015.01.006

Tracy, S.J., 2019, Qualitative research methods: Collecting evidence, crafting analysis, communicating impact, John Wiley \& Sons, Hoboken, NJ.

Ullah, F., Sepasgozar, S. \& Wang, C., 2018, 'A systematic review of smart real estate technology: Drivers of, and barriers to, the use of digital disruptive technologies and techno platforms', Sustainability 10(9), 3142. https://doi.org/10.3390/su10093142
online

Verkijika, S.F. \& De Wet, L., 2018, 'A usability assessment of e-government websites in Sub-Saharan Africa', International Journal of Information Management 39 (2018), 20-29. https://doi.org/10.1016/j.ijinfomgt.2017.11.003

Van den Heever, J. \& Rensburg, R., 2018, 'Investigating social media conversations: Towards implementing an online reputation management framework for NPOs', Communitas 23, 53-70. https://doi.org/10.18820/24150525/Comm.v23.4

Van Heerden, R., Von Solms, S. \& Vorster, J., 2018, 'Major security incidents since 2014: An African perspective', in 2018 IST-Africa Week Conference (IST-Africa), Gaborone, Botswana, May 9-11, 2018, pp. 1-11.

Ziakis, C., Vlachopoulou, M., Kyrkoudis, T. \& Karagkiozidou, M., 2019, 'Important factors for improving Google search rank', Future Internet 11(2), 32. https://doi. org/10.3390/fi11020032 